\title{
Adenosine A2b receptor promotes progression of human oral cancer
}

\author{
Hiroki Kasama', Yosuke Sakamoto², Atsushi Kasamatsu², Atsushi Okamoto', Tomoyoshi Koyama', \\ Yasuyuki Minakawa' ${ }^{1}$ Katsunori Ogawara², Hidetaka Yokoe ${ }^{3}$, Masashi Shiiba ${ }^{4}$, Hideki Tanzawa ${ }^{1,2}$ \\ and Katsuhiro Uzawa ${ }^{1,2^{*}}$
}

\begin{abstract}
Background: Adenosine A2b receptor (ADORA2B) encodes an adenosine receptor that is a member of the $G$ protein-coupled receptor superfamily. This integral membrane protein stimulates adenylate cyclase activity in the presence of adenosine. Little is known about the relevance of ADORA2B to human malignancy including oral squamous cell carcinoma (OSCC). We aimed to characterize the expression state and function of ADORA2B in OSCC.

Methods: The ADORA2B expression levels in nine OSCC-derived cells were analyzed by quantitative reverse transcriptase-polymerase chain reaction and immunoblotting analyses. Using an ADORA2B knockdown model, we assessed cellular proliferation and expression of hypoxia-inducible factorla (HIF-1a). We examined the adenosine receptor expression profile under both normoxic and hypoxic conditions in the OSCC-derived cells. In addition to in vitro data, the clinical correlation between the ADORA2B expression levels in primary OSCCS ( $n=100$ patients) and the clinicopathological status by immunohistochemistry (IHC) also was evaluated.
\end{abstract}

Results: $A D O R A 2 B$ mRNA and protein were up-regulated significantly $(p<0.05)$ in seven OSCC-derived cells compared with human normal oral keratinocytes. Suppression of ADORA2B expression with shRNA significantly $(p<0.05)$ inhibited cellular proliferation compared with the control cells. HIF-1a also was down-regulated in ADORA2B knockdown OSCC cells. During hypoxia, ADORA2B expression was induced significantly $(p<0.05)$ in the mRNA and protein after 24 hours of incubation in OSCC-derived cells. IHC showed that ADORA2B expression in primary OSCCs was significantly $(p<0.05)$ greater than in the normal oral counterparts and that ADORA2B-positive OSCCs were correlated closely $(p<0.05)$ with tumoral size.

Conclusion: Our results suggested that ADORA2B controls cellular proliferation via HIF-1a activation, indicating that ADORA2B may be a key regulator of tumoral progression in OSCCs.

\section{Background}

The adenosine receptors consists of four members that belong to the $\mathrm{G}$ protein-coupled receptor superfamily, including A1, A2A, A2B and A3 [1]. Activation of ADORA2A and ADORA2B causes activation of adenylate cyclase and phospholipase $\mathrm{C}$ [2]. ADORA2B is found in many different cellular types and requires higher concentrations of adenosine for activation than the

\footnotetext{
* Correspondence: uzawak@faculty.chiba-u.jp

'Department of Oral Science, Graduate School of Medicine, Chiba University, 1-8-1 Inohana, Chuo-ku, Chiba 260-8670, Japan

${ }^{2}$ Department of Dentistry and Oral-Maxillofacial Surgery, Chiba University

Hospital, 1-8-1 Inohana, Chuo-ku, Chiba 260-8670, Japan

Full list of author information is available at the end of the article
}

ADORA1, 2A, and 3 subtypes [2]. ADORA2B mainly is located peripherally and is involved in inflammation and immune response [3]. ADORA2B is not stimulated by physiologic levels of adenosine and therefore may play an important role in pathophysiologic conditions associated with massive adenosine release. Such conditions occur in tumors when hypoxia is commonly observed $[4,5]$. Moreover, ADORA2B was overexpressed in colorectal carcinomas grown under hypoxic conditions [6]. Adenosine was reported to modulate proliferation of cancer cells by a hypoxia-inducible factor $1 \alpha$ (HIF-1 $\alpha$ )dependent mechanism [7]. HIF-1 expression and activity also are regulated by important signal transduction pathways including those involving extracellular regulated 
kinase 1/2 (ERK1/2) and Akt [8]. We hypothesized that ADORA2B may be associated with cancer progression.

Growing evidence indicates that the adenosine-receptor pathway also may be a promising therapeutic target in a wide range of conditions, including cerebral and cardiac ischemic diseases, sleep disorders, immune and inflammatory disorders, and cancer [9]. However, the expression status and function of ADORA2B in oral squamous carcinoma (OSCC) have not been characterized fully.

Because the relationship between HIF- $1 \alpha$ and ADORA2B is just beginning to be understood, we assumed that dysregulation of ADORA2B is involved in progression of OSCCs via activation of HIF- $1 \alpha$. We also performed functional analysis to define the biologic effect of ADORA2B in OSCC.

\section{Methods}

\section{Ethics statement}

The Ethics Committee of the Graduate School of Medicine, Chiba University (approval number, 236) approved the study protocol, which was performed according to the tenets of the Declaration of Helsinki. All patients provided written informed consent.

\section{OSCC-derived cellular lines and tissue specimens}

Immortalized human OSCC-derived cell lines (HSC-2, HSC-3, HSC-4, Ca9-22, Sa3, HO-1-u-1, HO-1-N-1, $\mathrm{KOSC} 2$, and SAS) were obtained from the Human Science Research Resources Bank (Osaka, Japan) or the RIKEN BioResource Center (Ibaraki, Japan) through the National BioResource Project of the Ministry of Education, Culture, Sports, Science and Technology (Tokyo, Japan). Short tandem repeat profiles confirmed cellular identity. All OSCC-derived cells were grown in Dulbecco's modified Eagle medium/F-12 HAM (Sigma-Aldrich, St. Louis, MO, USA) supplemented with $10 \%$ fetal bovine serum (Sigma-Aldrich) and 50 units/ml penicillin and streptomycin (Sigma-Aldrich). Isolated and primarycultured human normal oral keratinocytes (HNOKs) were used as a normal tissue control [10-15]. They were isolated from healthy oral mucosal epithelial specimens from three young patients at Chiba University Hospital. The HNOKs were primary cultured and maintained in Keratinocyte-SFM (Invitrogen, Carlsbad, CA, USA) comprised of growth supplement (Invitrogen) and 50 units/ $\mathrm{ml}$ penicillin and streptomycin (Sigma).

One hundred pairs of primary OSCC samples and corresponding normal oral epithelial tissues were obtained during surgeries at Chiba University Hospital. The resected tissues were fixed in $20 \%$ buffered formaldehyde solution for pathologic diagnosis and immunohistochemistry (IHC). The Department of Pathology of Chiba University Hospital histopathologically diagnosed each OSCC sample according to the World Health
Organization criteria [16]. Clinicopathologic staging was determined by the TNM (tumor-node-metastasis) classification of the International Union against Cancer [17]. All patients had OSCC that was confirmed histologically; the samples were checked to ensure that tumoral tissue was present in more than $80 \%$ of the specimens.

\section{Preparation of CDNA and protein}

Total RNA was isolated using Trizol Reagent (Invitrogen) according to the manufacturer's instructions. cDNA was generated using $5 \mu \mathrm{g}$ total RNA from OSCC-derived cell lines using Ready-To-Go You-Prime First-Strand Beads (GE Healthcare, Buckinghamshire, UK) and oligo (dT) primer (Hokkaido System Science, Sapporo, Japan) according to the manufacturer's instructions. The cells were washed twice with cold phosphate-buffered saline (PBS) and centrifuged briefly. The cell pellets were incubated at $4{ }^{\circ} \mathrm{C}$ for 30 minutes in a lysis buffer $(7 \mathrm{M}$ urea, $2 \mathrm{M}$ thiourea, $4 \% \mathrm{w} / \mathrm{v}$ CHAPS, and $10 \mathrm{mM}$ Tris $\mathrm{pH} 7.4$ ) with a proteinase inhibitor cocktail (Roche Diagnostics, Mannheim, Germany). HNOKs proteins were extracted from the same samples obtained for RNA. The protein concentration was measured using the BCA Protein Assay Kit (Thermo, Rockford, IL, USA).

\section{mRNA expression analysis}

Real-time quantitative reverse transcriptase-polymerase chain reaction (qRT-PCR) was performed using the LightCycler 480 apparatus (Roche Diagnostics). Primers and universal probes were designed using the Universal Probe Library (Roche Diagnostics), which specifies the most suitable set. The primer sequences used for qRT-PCR were: ADORA2B, forward, 5 -GGGCTTCTGCACTGACTTCT -3'; reverse, 5' -CCGTGACCAAACTTTTATACCTG -3'; and universal probe \#34, and the glyceraldehyde-3phosphate dehydrogenase $(G A P D H)$, forward, 5 '-AGC CACATCGCTCAGACAC -3'; reverse, 5' -GCCCAATAC GACCAAATCC $-3{ }^{\prime}$; and universal probe \#60. The transcript amount for ADORA2B was estimated from the respective standard curves and normalized to the GAPDH transcript amount determined in corresponding samples. All samples were analyzed in triplicate, and three independent RNA preparations were analyzed from each cell line.

\section{Immunoblotting analysis}

Protein extracts $(20 \mu \mathrm{g})$ were separated by sodium dodecyl sulfate polyacrylamide gel electrophoresis in 4-12\% gel, transferred to nitrocellulose membranes, and blocked for 1 hour at room temperature in Blocking One (Nacalai Tesque, Tokyo, Japan). The membranes were incubated with rabbit anti-ADORA2B polyclonal antibody (Chemicon International, Temecula, CA, USA), mouse anti- $\alpha$ tubulin monoclonal antibody (Santa Cruz 
Biotechnology, Santa Cruz, CA, USA), mouse anti- HIF$1 \alpha$ monoclonal antibody (Santa Cruz Biotechnology), rabbit anti-Akt monoclonal antibody (Santa Cruz Biotechnology), rabbit anti-pAkt monoclonal antibody (Santa Cruz Biotechnology), rabbit anti-ERK1/2 monoclonal antibody (Santa Cruz Biotechnology), and rabbit anti-pERK1/2 monoclonal antibody (Santa Cruz Biotechnology) overnight at $4{ }^{\circ} \mathrm{C}$. The membranes were washed with $0.1 \%$ Tween-20 in Tris-buffered saline, incubated with secondary antibody and coupled to horseradish peroxidase-conjugated anti-rabbit or anti-mouse IgG (Promega, Madison, WI, USA) for 1 hour at room temperature. The membranes were detected using SuperSignal West Pico Chemiluminescent substrate (Thermo), and immunoblotting was visualized by exposing the membranes to ATTO Light-Capture II (ATTO, Tokyo, Japan). Signal intensities were quantitated using the CS Analyzer version 3.0 software (ATTO).

\section{Transfection with shRNA plasmid}

OSCC cellular lines (HSC-3 and -4) were transfected with ADORA2B shRNA (shADORA2B) or control shRNA (shMock) vectors (Santa Cruz Biotechnology) using Lipofectamine LTX and Plus Reagents (Invitrogen). After transfection, the stable transfectants were isolated by a culture medium containing $2 \mathrm{ng} / \mathrm{mL}$ Puromycin (Invitrogen). Two to three weeks after transfection, viable colonies were transferred to new dishes. shADORA2B and shMock cells were used for further experiments.

\section{Cellular growth}

The transfectants were seeded in six-well plates at a density of $1 \times 10^{4}$ viable cells/well. Cells were harvested for 168 hours and counted every 24 hours. We determined proliferation rates using the 3-(4,5-dimethylthiazol-2-yl)5-(3- carboxymethoxyphenyl)-2-(4-sulfophenyl)-2H-tetrazolium (MTS) assay (Promega).

\section{Apoptosis assay}

Apoptosis detection was performed in transfected and control cells by staining with the Annexin V-FITC Apoptosis Detection kit (Abcam, Cambridge, MA, USA). Cells were harvested at a density of $2 \times 10^{5}$ cells $/ \mathrm{ml}$ in $1 \times$ binding buffer and stained with FITC-labeled Annexin V and propidium iodide (PI) for $5 \mathrm{~min}$ at room temperature. Samples were immediately analyzed using the FACScaliber flow cytometer (Becton-Dickinson, San Jose, CA, USA).

\section{Hypoxic treatment}

To create hypoxia, cells were placed for 12 to 48 hours in a modular incubator chamber (Astec, Fukuoka, Japan) and flushed with a gas mixture containing $1 \%$ oxygen, $5 \%$ carbon dioxide, and balance N2.
IHC

IHC was performed on 4- $\mu \mathrm{m}$ sections of paraffinembedded specimens using rabbit anti-ADORA2B polyclonal antibody (Chemicon International) and mouse anti- HIF-1 $\alpha$ monoclonal antibody (Santa Cruz Biotechnology). Briefly, after deparaffinization and hydration, the endogenous peroxidase activity was quenched by a 30-minute incubation in a mixture of $0.3 \%$ hydrogen peroxide solution in $100 \%$ methanol; the sections were blocked for 2 hours at room temperature with $1.5 \%$ blocking serum (Santa Cruz Biotechnology) in PBS before reacting with anti-ADORA2B antibody overnight at $4{ }^{\circ} \mathrm{C}$ in a moist chamber. Upon incubation with the

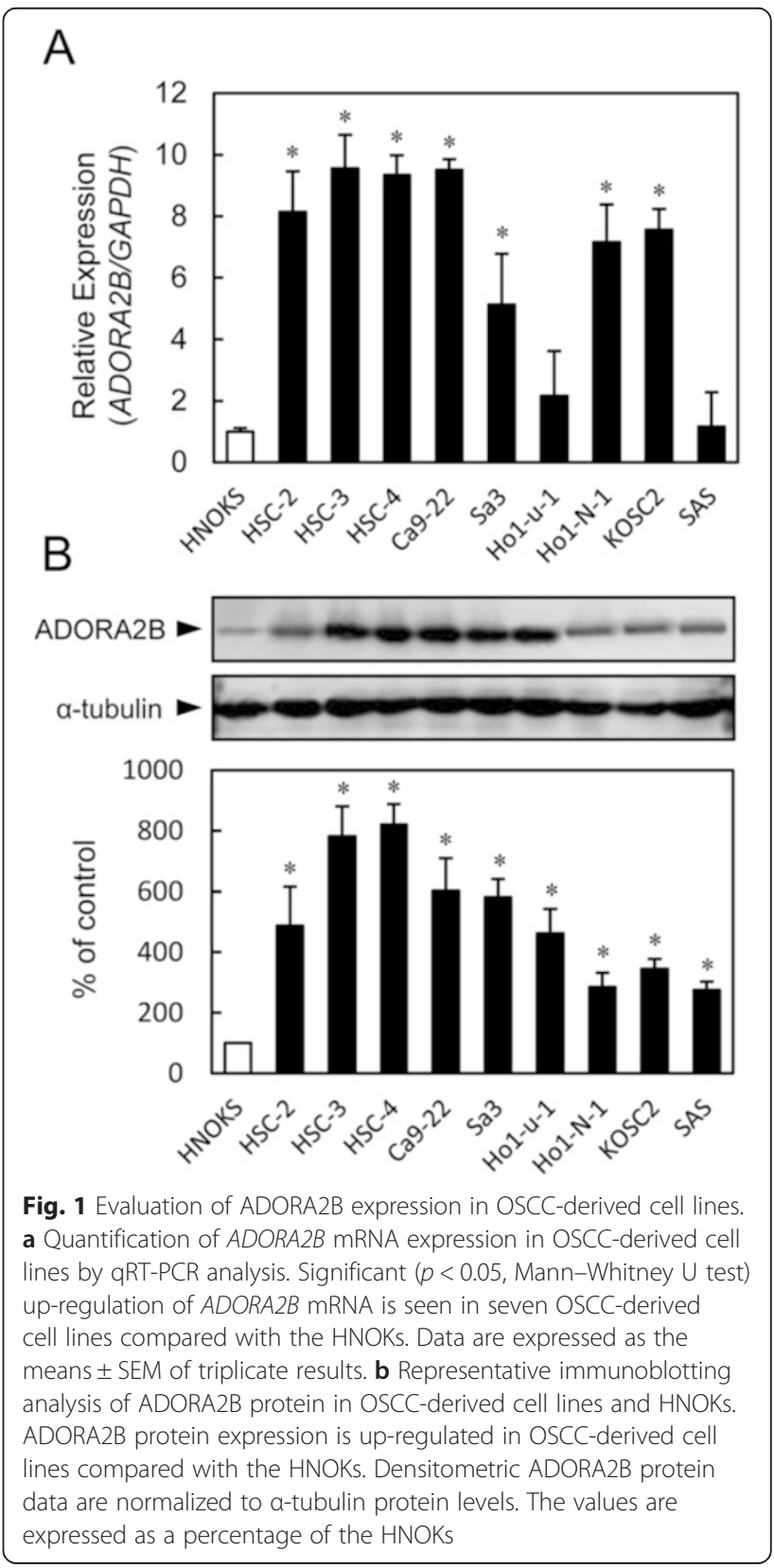


primary antibody, the specimens were washed three times in PBS and treated with Envision reagent (DAKO, Carpinteria, CA, USA) followed by color development in 3,3' -diaminobenzidine tetrahydrochloride (DAKO). The slides then were counterstained slightly with hematoxylin, dehydrated with ethanol, cleaned with xylene, and mounted. Nonspecific binding of an antibody to proteins other than the antigen sometimes occurred. As a negative control, triplicate sections were immunostained without exposure to primary antibodies, which confirmed the staining specificity. To quantify the status of ADORA2B protein expression in those components, we used the IHC scoring systems described previously [13, 15, 18-20]. The mean percentages of positive tumoral cells were determined in at least three random fields at 400× magnification in each section. The intensities of the ADORA2B immunoreactions were scored as follows: $0+$, none; $1+$, weak; $2+$, moderate; and $3+$, intense. The cellular number
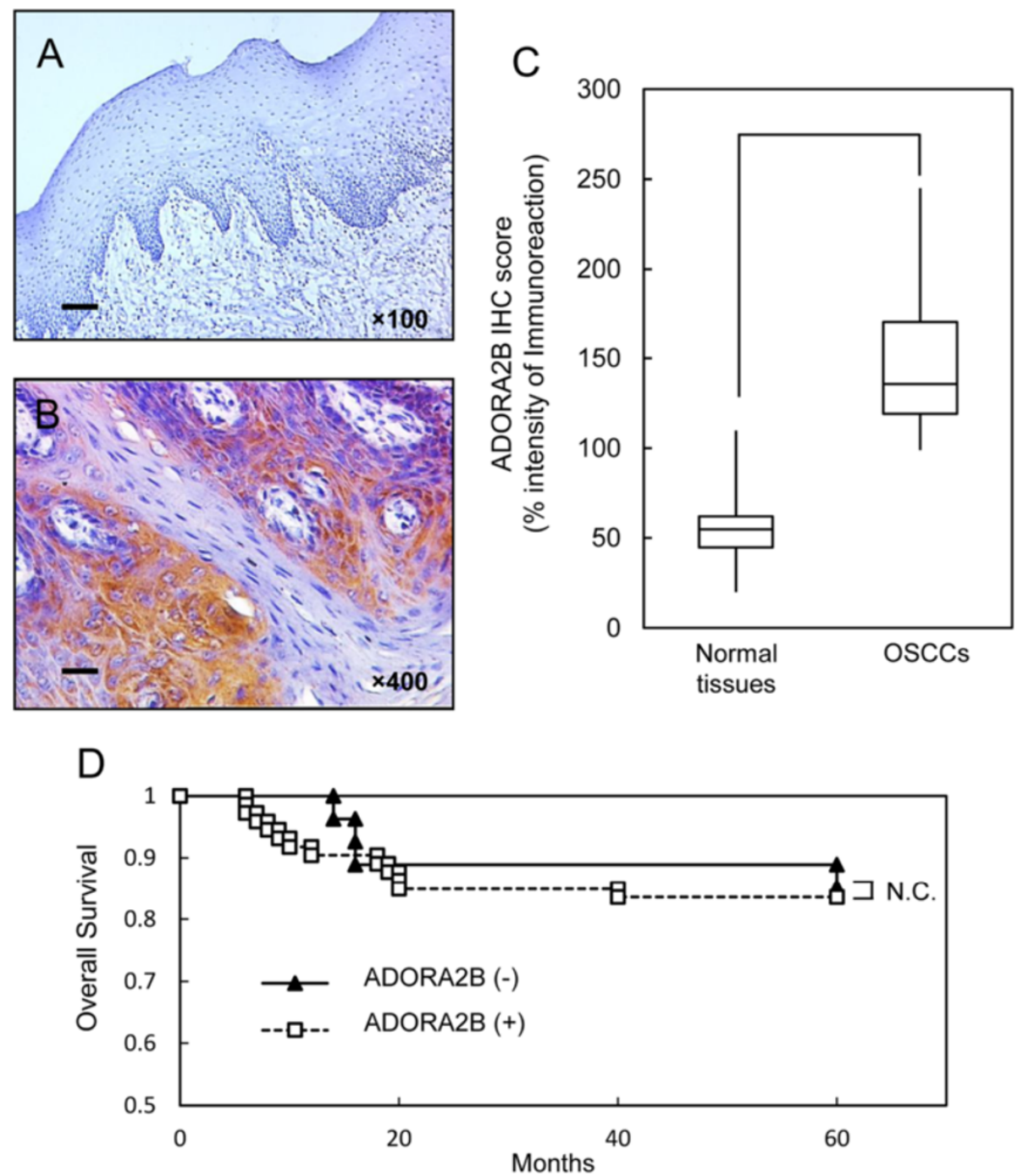

Fig. 2 Evaluation of ADORA2B protein expression in primary OSCCS. $\mathbf{a}, \mathbf{b}$ Representative $I H C$ results of ADORA2B in normal oral tissue and primary OSCC. a Normal oral tissues show almost negative immunostaining. Original magnification, $\times 100$. Scale bars, 50 mm. b ADORA2B-positive cases of OSCC. Positive immunoreaction for ADORA2B is detected in the cytoplasm. Original magnification, $\times 400$. Scale bars, $10 \mathrm{~mm}$. c The status of ADORA2B protein expression in primary OSCCS $(n=100)$ and the normal counterparts based on an IHC scoring system. $I H C$ scores are calculated as follows: $I H C$ score $=1 \times$ (number of weakly stained cells in the field) $+2 \times$ (number of moderately stained cells in the field) $+3 \times$ (number of intensely stained cells in the field). The ADORA2B IHC scores for normal oral tissues and OSCCs range from 20 to 110 (median, 51) and 99 to 245 (median, 139.5), respectively. ADORA2B protein expression levels in OSCCs are significantly $(p<0.05$, Mann-Whitney $U$ test) higher than in normal oral tissues. $\mathbf{d}$ Kaplan-Meier survival curve for overall survival rates of patients with OSCCs based on the levels of ADORA2B expression. Up-regulated ADORA2B expression was not significantly related to overall survival rates (N.S. $=p=0.8037$ ). The log-rank statistic was used to test the difference in survival times between the groups. ADORA2B $(+)$, up-regulated ADORA2B; ADORA2B (-), down-regulated ADORA2B 
and staining intensity were multiplied to produce an ADORA2B IHC score. Cases with an ADORA2B IHC score exceeding 120.0 ( +3 standard deviation [SD] score for normal tissue) were defined as ADORA2B-positive. The \pm 3 -SD cutoff, which statistically is just $0.2 \%$ of the measurement and would be expected to fall outside this range, was used because it was unlikely to be affected by random experimental error produced by sample manipulation [21]. Two independent pathologists from Chiba University Hospital, neither of whom had knowledge of the patients' clinical status, made these judgments.

\section{Statistical analysis}

In comparisons of ADORA2B expression levels, statistical significance was evaluated using the Mann-Whitney $U$ test. Relationships between ADORA2B-immunohistochemical staining scores and clinicopathological profiles were evaluated using the $\mathrm{x} 2$ test, Fisher's exact test, and Mann-Whitney $\mathrm{U}$ test. $p<0.05$ was considered significant. The data are expressed as the mean \pm standard error of the mean (SEM). Survival curves were obtained by the Kaplan-Meier method, and differences in survival rates between ADORA2B-positive and ADORA2B negative cases were compared by logrank test with $95 \%$ significance.

\section{Results}

\section{Evaluation of ADORA2B expression in OSCC-derived cell} lines

To analyze the expression status of ADORA2B, we performed qRT-PCR and immunoblotting analysis using nine OSCC-derived cell lines (HSC-2, HSC-3, HSC-4, Cap-22, Sa3, Ho-1-u-1, Ho-1-N-1, KOSC2, and SAS) and HNOKs. ADORA2B mRNA was up-regulated significantly $(p<0.05)$ in seven OSCC-derived cell lines compared with the HNOKs (Fig. 1a). Figure 1b shows representative results of immunoblotting analysis. ADORA2B protein expression increased significantly in all OSCCderived cell lines compared with the HNOKs. Expression analysis indicated that both transcription and translation products of this molecule were highly expressed in the OSCC-derived cell lines.

\section{Evaluation of ADORA2B expression in primary OSCCs}

To determine the expression status of ADORA2B in primary OSCCs and the relation to the clinicopathological characteristics, we investigated ADORA2B protein expression in primary OSCCs and paired normal oral tissues from 100 patients using the IHC scoring system. Representative IHC results for ADORA2B protein in the normal oral tissue and primary OSCC are shown in Fig. 2a-c. Positive staining was seen predominantly in the cytoplasm of primary OSCC samples (Fig. 2b). The ADORA2B IHC scores in primary OSCCs (range, 99-
245; median, 139.5) were significantly higher than in normal tissues (20-110; median, 51) (Fig. 2c) $(p<0.05)$.

Table 1 shows the correlations between the clinicopathological characteristics of the patients with OSCC and the status of ADORA2B protein expression using the IHC scoring system. We defined cases with an IHC score exceeding 120 (+3-SD score for normal tissue) as ADORA2B-positive. Among the clinical parameters, ADORA2B expression was related significantly $(p=0.02)$

Table 1 Correlation between ADORA2B expression and clinical classification

\begin{tabular}{|c|c|c|c|c|}
\hline \multirow{3}{*}{$\begin{array}{l}\text { Clinical } \\
\text { classification }\end{array}$} & \multirow[t]{3}{*}{ Total } & \multirow{2}{*}{\multicolumn{2}{|c|}{$\begin{array}{l}\text { Results of immunostaining } \\
\text { No. patients (\%) }\end{array}$}} & \multirow[t]{3}{*}{$p$ value } \\
\hline & & & & \\
\hline & & $\begin{array}{l}\text { ADORA2B } \\
\text { negative }\end{array}$ & $\begin{array}{l}\text { ADORA2B } \\
\text { positive }\end{array}$ & \\
\hline \multicolumn{5}{|c|}{ Age at surgery (years) } \\
\hline$<60$ & 27 & $7(26)$ & $20(74)$ & \multirow[t]{3}{*}{0.95} \\
\hline$\geqq 60,<70$ & 26 & $8(31)$ & $18(69)$ & \\
\hline$\geqq 70$ & 47 & $13(28)$ & $34(72)$ & \\
\hline \multicolumn{5}{|l|}{ Gender } \\
\hline Male & 64 & $21(33)$ & $43(67)$ & \multirow[t]{2}{*}{0.24} \\
\hline Female & 36 & $7(19)$ & $29(81)$ & \\
\hline \multicolumn{5}{|l|}{ T-primary tumor } \\
\hline $\mathrm{T} 1$ & 8 & $3(38)$ & $5(62)$ & \multirow[t]{4}{*}{$0.02^{*}$} \\
\hline $\mathrm{T} 2$ & 60 & $22(37)$ & $38(63)$ & \\
\hline T3 & 15 & $2(13)$ & $13(87)$ & \\
\hline T4 & 17 & $1(6)$ & $16(94)$ & \\
\hline $\mathrm{T} 1+\mathrm{T} 2$ & 58 & $25(43)$ & $33(57)$ & \multirow[t]{2}{*}{$0.01^{*}$} \\
\hline $\mathrm{T} 3+\mathrm{T} 4$ & 32 & $3(10)$ & $29(90)$ & \\
\hline \multicolumn{5}{|c|}{ N-regional lymph node } \\
\hline$N$ (negative) & 58 & $14(24)$ & $44(76)$ & \multirow[t]{2}{*}{0.39} \\
\hline N (positive) & 42 & $14(33)$ & $28(67)$ & \\
\hline \multicolumn{5}{|l|}{ Stage } \\
\hline I & 7 & $3(43)$ & $4(57)$ & \multirow[t]{4}{*}{0.54} \\
\hline$\|$ & 38 & $11(29)$ & $27(71)$ & \\
\hline III & 17 & $4(24)$ & $13(76)$ & \\
\hline IV & 38 & $10(26)$ & $28(74)$ & \\
\hline \multicolumn{5}{|c|}{ Histopathologic type } \\
\hline Well & 64 & $15(23)$ & $49(77)$ & \multirow[t]{3}{*}{0.25} \\
\hline Moderately & 31 & $11(35)$ & $20(65)$ & \\
\hline Poorly & 5 & $2(40)$ & $3(60)$ & \\
\hline \multicolumn{5}{|l|}{ Tumor site } \\
\hline Gingiva & 35 & $11(31)$ & $24(69)$ & \multirow[t]{4}{*}{0.84} \\
\hline Tongue & 50 & $12(24)$ & $38(76)$ & \\
\hline Buccal mucosa & 9 & $2(22)$ & $7(78)$ & \\
\hline Oral floor & 6 & $3(50)$ & $3(50)$ & \\
\hline
\end{tabular}


to the primary size of the OSCC tumors. Moreover, a significant $(p=0.01)$ difference was found in the ADORA2B expression levels between the T1/T2 group and the T3/T4 group. Survival analysis using the Kaplan-Meier method showed that ADORA2B expression was not significantly related to overall survival ( $P=0.8037$; Fig. $2 \mathrm{~d})$.

\section{Establishment of ADORA2B knockdown cells}

The OSCC-derived cell lines (HSC-3 and -4) were transfected with ADORA2B shRNA (shADORA2B) and the control shRNA (shMock). qRT-PCR and immunoblotting analyses were performed to assess the efficiency of ADORA2B knockdown. ADORA2B mRNA expression in
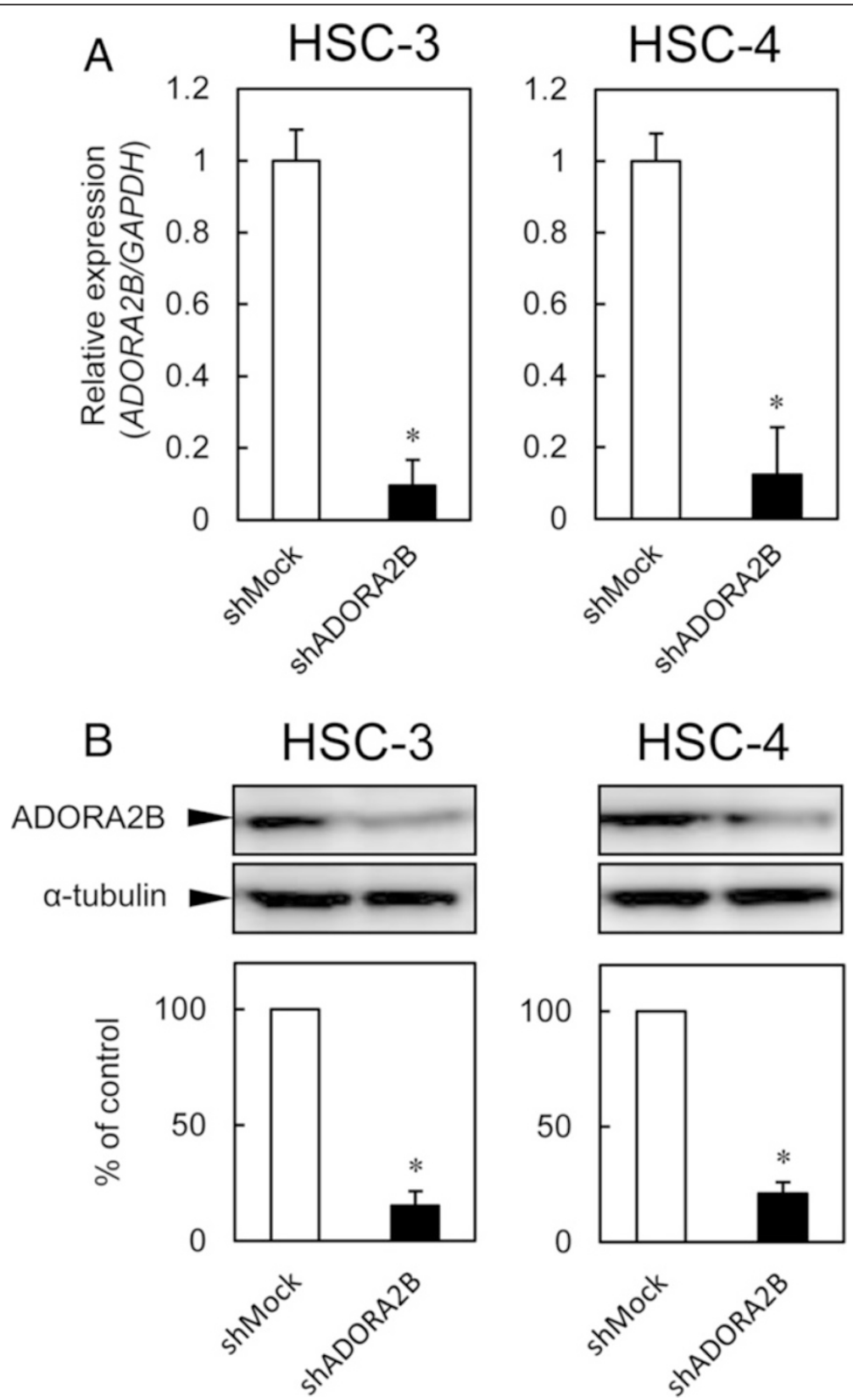

Fig. 3 Expression of ADORA2B in shADORA2B-transfected cells. a Expression of ADORA2B mRNA in shMock- and shADORA2B-transfected cells (HSC-3- and -4-derived transfectants). The ADORA2B mRNA expression in the shADORA2B is significantly ( $p<0.05$, Mann-Whitney $U$ test) lower than that in the shMock-transfected cells. $\mathbf{b}$ Immunoblotting analysis of ADORA2B protein in the shMock- and shADORA2B-transfected cells (HSC-3- and -4-derived transfectants). The ADORA2B protein in the shADORA2B transfected cells is decreased markedly compared with the shMock-transfected cells 
shADORA2B-transfected cells was significantly $(p<0.05)$ lower than in shMock-transfected cells (Fig. 3).

\section{Reduced cellular growth in ADORA2B knockdown cells}

To study the effect of ADORA2B knockdown on cellular growth, we performed a cellular proliferation assay. shADORA2B and shMock cells were seeded in six-well plates at a density of $1 \times 10^{4}$ viable cells/well that were counted for 168 hours. Both HSC-3 and -4 shADORA2B cells had a significant $(p<0.05)$ decrease in cellular growth compared with the shMock cells (Fig. 4a). To confirm apoptotic activity, shADORA2B and shMock cells were analyzed for Annexin V expression by flow cytometry. There was no significant difference in the percentage of annexin-
$\mathrm{V}$ and PI positive cells in the shADORA2B and shMock cells (Fig. 4b).

\section{Hypoxic induction of ADORA2B}

To investigate the relationship between ADORA2B expression and oxygen supply, we examined ADORA2B expression under both normoxic and hypoxic conditions in the OSCC-derived cell lines (HSC-3 and -4, with higher ADORA2B protein expression). The expression of ADORA2B mRNA and protein were up-regulated significantly $(p<0.05)$ in these cell lines after 24 hours of hypoxia compared with a normoxia (Fig. 5a, b). Immunocytofluorescence showed that the ADORA2B

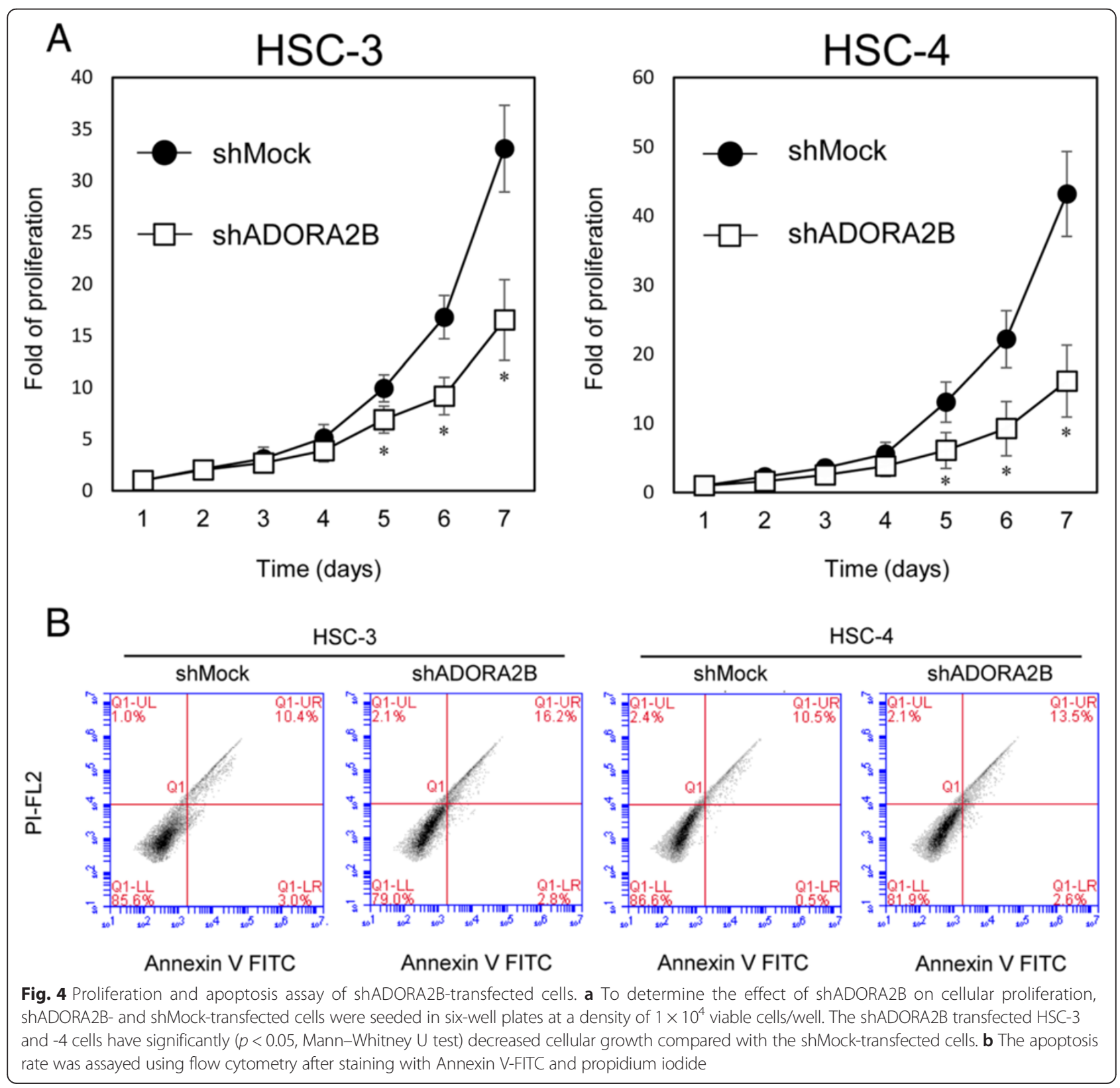



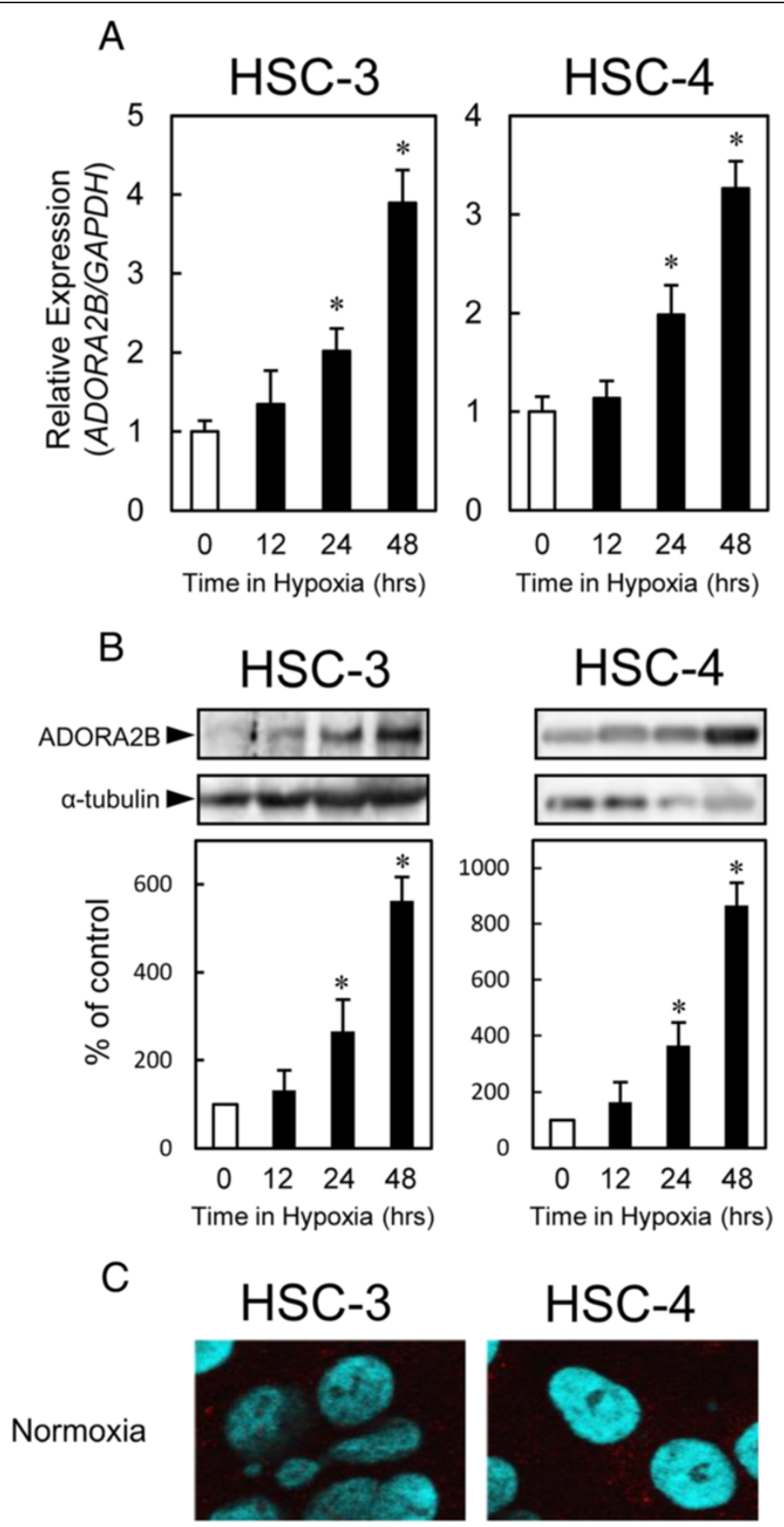

Hypoxia
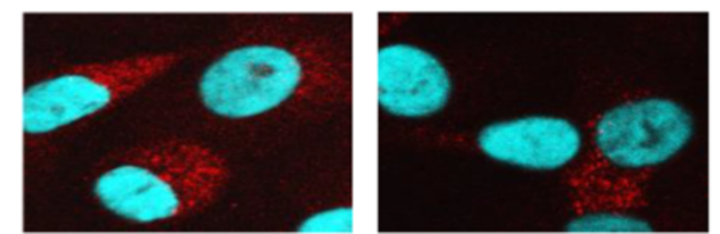

Fig. 5 (See legend on next page.) 
(See figure on previous page.)

Fig. 5 Hypoxic induction of ADORA2B. a OSCC-derived cell lines are exposed to hypoxia (1 \% oxygen ) and probed for ADORA2B expression by qRT-PCR. Expression of ADORA2B mRNA is induced significantly ( $p<0.05$, Mann-Whitney $U$ test) by hypoxia in OSCC-derived cell lines (HSC-3 and -4). $\mathbf{b}$ Immunoblotting analysis shows that the ADORA2B protein levels in hypoxia also have increased markedly compared with that in normoxia. c Immunocytofluorescence labeling of ADORA2B (red). Cellular nuclei are labeled with DAPI (blue). Immunocytofluorescence shows that the ADORA2B expression levels are higher in hypoxia than in normoxia in OSCC-derived cell lines (HSC-3 and -4)

expression levels were higher in hypoxia than in normoxia in these cell lines (Fig. 5c).

\section{Activation of HIF-1a}

To investigate a potential underlying mechanism of reduced cellular proliferation in the shADORA2Btransfected cells, we assessed the HIF- $1 \alpha$ pathways. ADORA2B knockdown causes markedly decreased protein expression levels of HIF-1 $\alpha$ compared with shMock-transfected cells (Fig. 6). To evaluate the status of the protein expressions of ERK1/2 phosphorylation (pERK1/2) and Akt phosphorylation (pAkt), we performed immunoblotting analyses in the shADORA2B transfected cells. ADORA2B knockdown caused markedly decreased levels of pERK1/2 and pAkt compared with shMock-transfected cells. Results from immunoblotting analyses indicated that the phosphorylation levels of ERK1/2 and Akt decreased, whereas ERK1/2 and Akt proteins were unchanged (Fig. 6). Moreover, to evaluate the status of the protein expressions of HIF- $1 \alpha$, pERK1/2, and pAkt under hypoxic conditions, we performed immunoblotting analyses in the shMock cells and shADORA2B-transfected cells. HIF- $1 \alpha$, pERK1/2, and pAkt expression levels were higher in hypoxia than in normoxia. During a hypoxic conditions, the levels of HIF-1 $\alpha$, pERK1/2, and pAkt protein were not increased significantly in the shADORA2B-transfected cells (Fig. 6). In addition to the in vitro data, we also investigated the correlation of ADORA2B and HIF- $1 \alpha$ in ADORA2Bpositive clinical samples by IHC staining. The high expression of HIF- $1 \alpha$ protein was detected in 19 out of 20 these samples (Additional file 1).

\section{Discussion}

There is growing evidence that adenosine receptor agonists are attractive therapeutic targets for a number of conditions, including inflammation and certain cancer types [1, 22-27]. Adenosine receptors are found to be up-regulated in various tumoral cells [28]. ADORA2B has important roles in cellular proliferation and differentiation and apoptosis of tumoral cells [29-31]. Our previous microarray data showed significant up-regulation of ADORA2B in OSCC-derived cell lines [32]. The current study found a high prevalence of increased ADORA2B expression in patients with OSCC and a significant correlation with the primary tumoral size (Table 1 ). These findings indicated that ADORA2B overexpression may be linked to human oral carcinogenesis, has an important role in OSCC development, and is related closely to tumoral progression. However, the detailed function of ADORA2B in OSCC remains unclear. To determine whether ADORA2B function is relevant to OSCC progression, we performed functional analysis to define the biologic effect of ADORA2B.

A significant up-regulation of ADORA2B was detected in all OSCC-derived cells examined at protein levels (Fig. 1b), indicating that ADORA2B expression is necessary for oral carcinogenesis. On the other hand, the mRNA expression states depended on the cells (Fig. 1a). A possible explanation for this discrepancy is that ADORA2B proteins are differently affected by the posttranslational ubiquitination and proteolysis in each tumor cell lines. In this context, discrepancies between mRNA expression levels and protein levels has been reported in OSCC cellular lines [33]. Many studies have indicated that ADORA2B plays critical roles, including tumoral development and progression, in cancers [3, 5-7, 31, 32]. In contrast, some studies have reported that ADORA2B has no role in adenosine-induced proliferation of human melanoma cells [34]. ADORA2B was up-regulated consistently in colorectal carcinoma tissues and colon cancer cell lines compared with normal colorectal mucosa [6].

The current study showed that hypoxia was involved with ADORA2B up-regulation in OSCC cells (Fig. 5a, b). Most solid tumors do not receive sufficient oxygen and tumoral cells grow in hypoxia [6]. Hypoxia promotes release of vascular endothelial growth factor by increasing ADORA2B expression and decreasing ADORA2A expression in human endothelial and smooth muscle cells [35].

Intratumoral hypoxia and genetic alterations lead to HIF- $1 \alpha$ overexpression, which has been associated with increased patient mortality in several cancer types [36]. To date, expression of ADORA2B correlation with HIF- $1 \alpha$ expression have not been elucidated in OSCC tissues. Our data showed that the immunoexpression of ADORA2B was correlated with HIF- $1 \alpha$ expression in primary OSCCs (Additional file 1). HIF-1 $\alpha$ is a common transcription factor induced by ADORA2B activation in inflammatory bowel disease and urologic diseases [37, 38]. Based on these findings, we observed the expression status of HIF$1 \alpha$ in the ADORA2B knockdown cells. As expected, HIF$1 \alpha$ was down-regulated significantly in the ADORA2B knockdown cells. We assessed the ERK1/2 and Akt pathways, which are up-regulated frequently in other cancers 

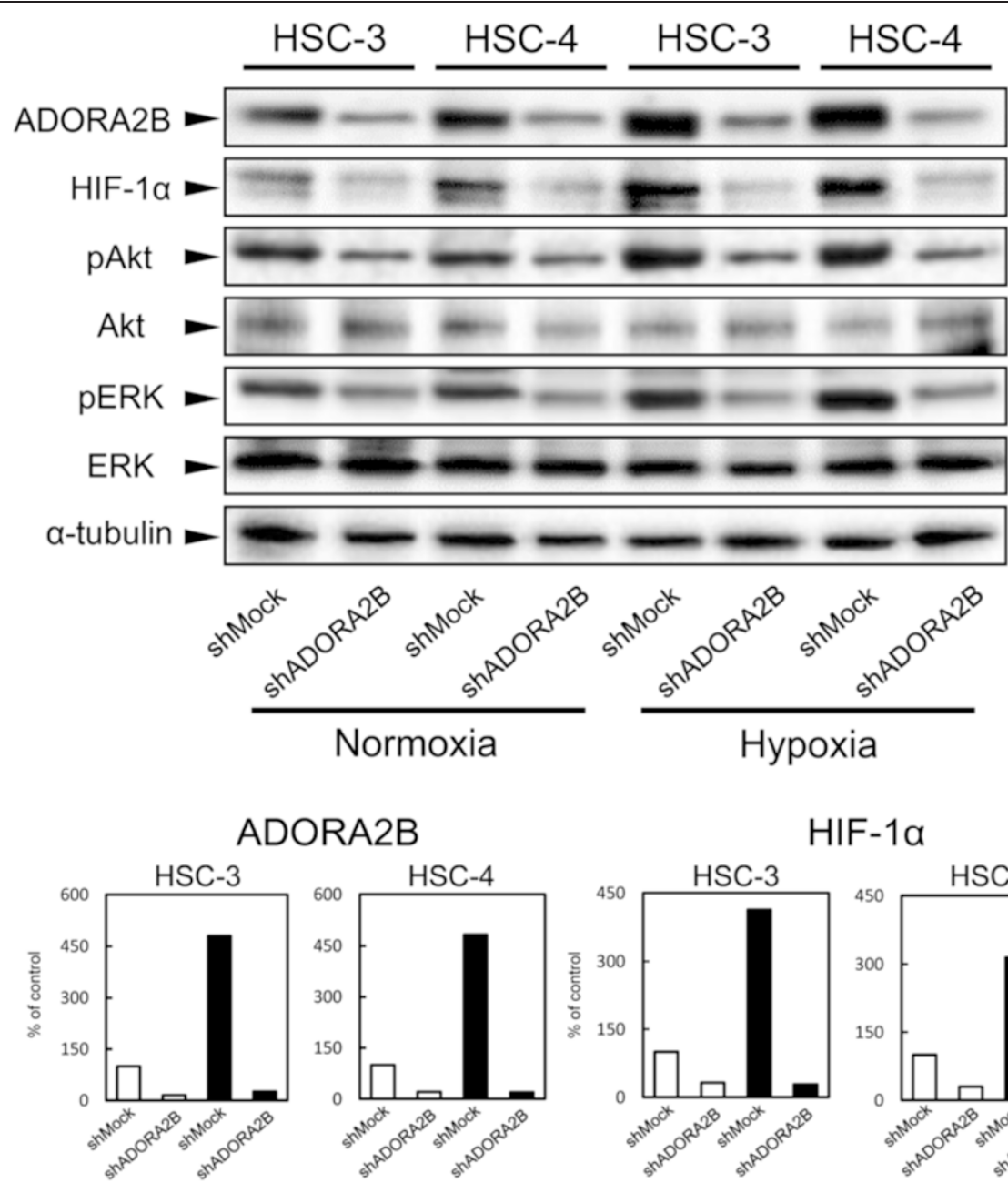

HIF-1a

ADORA2B
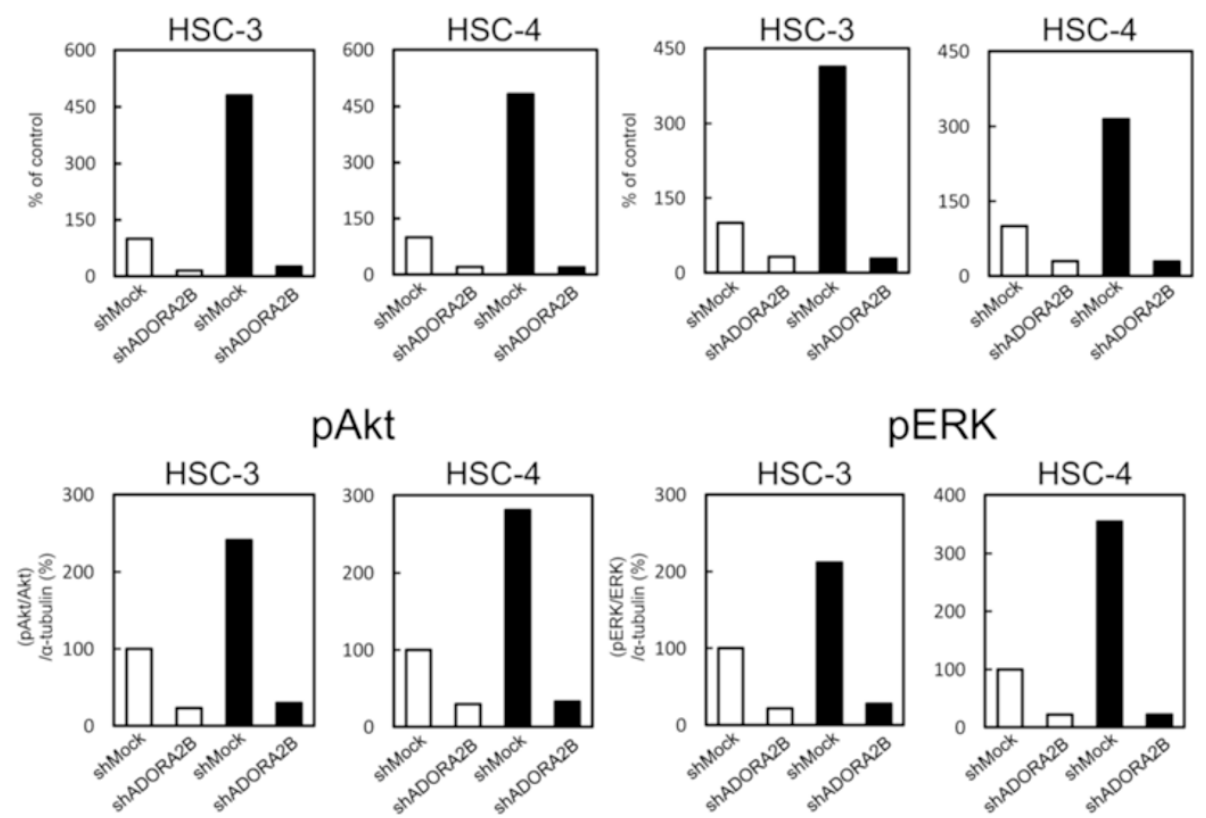

pAkt

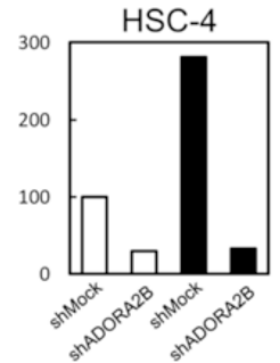

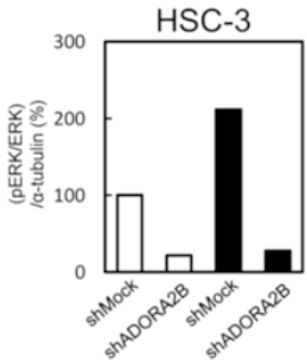

Normoxia

pERK

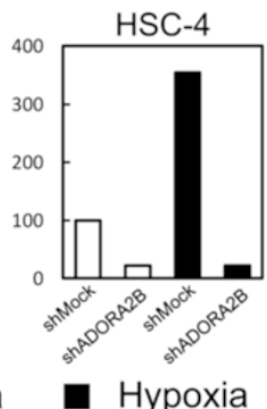

Fig. 6 ADORA2B knockdown inhibits HIF-1a, Akt, and ERK1/2 activation. Immunoblotting analysis of ADORA2B, HIF-1a, pERK1/2, and pAkt proteins in the Mock and shADORA2B cells under both normoxic and hypoxic conditions. The expression of ADORA2B, HIF-1a, pERK1/2, and pAkt protein were up-regulate in the shMock cells of hypoxia compared with a normoxia. The HIF-1a protein levels in the shADORA2B-transfected cells (HSC-3- and -4derived transfectants) also have decreased markedly compared with that in the shMock-transfected cells under both normoxic and hypoxic conditions. ADORA2B knockdown causes decreased levels of pAkt and pERK1/2 compared with the shMock-transfected cells (HSC-3-and -4-derived transfectants); the AKT and ERK1/2 protein levels are unchanged under both normoxic and hypoxic conditions. Densitometric ADORA2B, HIF-1a, pAkt/Akt and pERK ERK protein data were normalized to the a-tubulin protein levels

[7, 39], through HIF- $1 \alpha$ activity and found that the phosphorylation levels of ERK1/2 and Akt decreased, whereas ERK1/2 and Akt proteins were unchanged. Moreover, results from immunoblotting analyses indicated that hypoxia promotes the up-regulation of HIF- $1 \alpha$, pERK $1 / 2$, and pAkt by increasing ADORA2B expression (Fig. 6). ERK1/2 
and Akt activities are required by ADORA2B receptor activation [7]. The ADORA2B antagonist MRE 2029 F20 reduced ERK1/2 and Akt phosphorylation levels in melanoma cells in hypoxia [39].

\section{Conclusion}

The current results indicated that ADORA2B is overexpressed frequently in OSCCs and may be associated strongly with tumoral progression. During hypoxia, there was significant up-regulation of ADORA2B expression in OSCC-derived cell lines. While further studies are needed to determine the interaction between ADORA2B and HIF- $1 \alpha$, the current data indicated that ADORA2B may be an efficacious treatment target in progression of primary OSCCs.

\section{Additional file}

Additional file 1: Evaluation of ADORA2B and HIF-1a expression in primary OSCCs. (A-D) Representative $I H C$ results of $A D O R A 2 B$ and HIF-1a in the same OSCC samples. (A, B) ADORA2B-positive cases of OSCC. Positive immunoreaction for ADORA2B is detected in the cytoplasm. Original magnification, $\times 100$ (A) and $\times 400$ (C). (C, D) HIF-1a-positive cases of OSCC. Positive immunoreaction for HIF-1a is detected in the nuclear and cytoplasm. Original magnification, $\times 100$ (C) and $\times 400$ (D). (TIFF 4229 kb)

\section{Abbreviations}

ADORA2B: Adenosine A2b receptor; ERK1/2: Extracellular regulated kinase 1/ 2; HIF-1a: Hypoxia-inducible factor1a; HNOKs: Human normal oral keratinocytes; IHC: Immunohistochemistry; OSCC: Oral squamous cell carcinoma; qRT-PCR: Real-time quantitative reverse transcriptase-polymerase chain reaction.

\section{Competing interests}

The authors declare that they have no competing interests.

\section{Authors' contributions}

Conceived and designed the experiments: HK, YS, HT, KU. Performed the experiments: HK, YS, KU. Analyzed the data: HK, YS, KU. Contributed reagents/ materials/analysis tools: HK, YS, AK, AO, TK, YM, KO, HY, MS, HT, KU. Wrote the paper: HK, YS, HT, KU. All authors read and approved the final manuscript.

\section{Acknowledgments}

We thank Ms. Lynda C. Charters for editing this manuscript. The author received no financial support.

\section{Author details \\ 'Department of Oral Science, Graduate School of Medicine, Chiba University, 1-8-1 Inohana, Chuo-ku, Chiba 260-8670, Japan. ${ }^{2}$ Department of Dentistry and Oral-Maxillofacial Surgery, Chiba University Hospital, 1-8-1 Inohana, Chuo-ku, Chiba 260-8670, Japan. ${ }^{3}$ Department of Oral and Maxillofacial Surgery Research Institute, National Defense Medical College Hospital, 3-2, Namiki, Tokorozawa-shi, Saitama 359-8513, Japan. ${ }^{4}$ Department of Medical Oncology, Graduate School of Medicine, Chiba University, 1-8-1 Inohana, Chuo-ku, Chiba 260-8670, Japan.}

Received: 17 September 2014 Accepted: 27 July 2015

Published online: 31 July 2015

\section{References}

1. Gao ZG, Jacobson KA. Emerging adenosine receptor agonists. Expert Opin Emerg Drugs. 2007;12:479-92.
2. Linden J. Molecular approach to adenosine receptors: receptormediated mechanisms of tissue protection. Annu Rev Pharmacol Toxicol. 2001;41:775-87

3. Merighi S, Mirandola P, Varani K, Gessi S, Leung E, Baraldi PG, et al. A glance at adenosine receptors: novel target for antitumor therapy. Pharmacol Ther. 2003;100:31-48

4. Illes P, Klotz K-N, Lohse MJ. Signaling by extracellular nucleotides and nucleosides. Naunyn-Schmiedebergs Arch Pharmacol. 2000;362:295-8.

5. Fredholm BB, Irenius E, Kull B, Schulte G. Comparison of the potency of adenosine as an agonist at human adenosine receptors expressed in Chinese hamster ovary cells. Biochem Pharmacol. 2001;61:443-8.

6. Ma DF, Kondo T, Nakazawa T, Niu DF, Mochizuki K, Kawasaki T, et al. Hypoxia-inducible adenosine $\mathrm{A} 2 \mathrm{~B}$ receptor modulates proliferation of colon carcinoma cells. Hum Pathol. 2010;41:1550-7.

7. Merighi S, Benini A, Mirandola P, Gessi S, Varani K, Simioni C, et al. Caffeine inhibits adenosine-induced accumulation of hypoxia-inducible factor1alpha, vascular endothelial growth factor, and interleukin-8 expression in hypoxic human colon cancer cells. Mol Pharmacol. 2007;72:395-406.

8. Semenza GL. Expression of hypoxia-inducible factor 1: mechanisms and consequences. Biochem Pharmacol. 2002;64:993-8.

9. Jacobson KA, Gao ZG. Adenosine receptors as therapeutic targets. Nat Rev Drug Discov. 2006:5:247-64.

10. Endo Y, Uzawa K, Mochida Y, Shiiba M, Bukawa H, Yokoe H, et al. Sarcoendoplasmic reticulum $\mathrm{Ca}(2+)$ ATPase type 2 downregulated in human oral squamous cell carcinoma. Int J Cancer. 2004;110:225-31.

11. Kasamatsu A, Uzawa K, Nakashima D, Koike H, Shiiba M, Bukawa H, et al. Galectin-9 as a regulator of cellular adhesion in human oral squamous cell carcinoma cell lines. Int J Mol Med. 2005;16:269-73.

12. Koike H, Uzawa K, Grzesik WJ, Seki N, Endo Y, Kasamatsu A, et al. GLUT1 is highly expressed in cementoblasts but not in osteoblasts. Connect Tissue Res. 2005:46:117-24.

13. Sakuma K, Kasamatsu A, Yamatoji M, Yamano Y, Fushimi K, lyoda M, et al Expression status of Zic family member 2 as a prognostic marker for ora squamous cell carcinoma. J Cancer Res Clin Oncol. 2010;136:553-9.

14. Shimizu T, Kasamatsu A, Yamamoto A, Koike K, Ishige S, Takatori H, et al. Annexin A10 in human oral cancer: biomarker for tumoral growth via G1/S transition by targeting MAPK signaling pathways. PLoS One. 2012;6, e45510.

15. Yamatoji M, Kasamatsu A, Kouzu Y, Koike H, Sakamoto Y, Ogawara K, et al. Dermatopontin: a potential predictor for metastasis of human oral cancer. Int J Cancer. 2012;130:2903-11.

16. Pindborg JJ, Reichart PA, Smith CJ, Waal I. World Health Organization classification of tumours. Histological typing of cancer and precancer of the oral mucosa. 2nd ed. Berlin: Springer; 1997.

17. Sobin LH, Gospodarowicz MK, Wittekind C. TNM classification of malignant tumors. 7th ed. New York: Wiley-Liss; 2009.

18. Shimada K, Uzawa K, Kato M, Endo Y, Shiiba M, Bukawa H, et al. Aberrant expression of RAB1A in human tongue cancer. $\mathrm{Br} J$ Cancer. 2005;92:1915-21.

19. Kouzu Y, Uzawa K, Koike H, Saito K, Nakashima D, Higo M, et al. Overexpression of stathmin in oral squamous-cell carcinoma: correlation with tumour progression and poor prognosis. Br J Cancer. 2006;94:717-23.

20. Iyoda M, Kasamatsu A, Ishigami T, Nakashima D, Endo-Sakamoto Y, Ogawara $\mathrm{K}$, et al. Epithelial cell transforming sequence 2 in human oral cancer. PLoS One. 2010;5, e14082

21. Verburg FA, Wäschle K, Reiners C, Giovanella L, Lentjes EG. Heterophile antibodies rarely influence the measurement of thyroglobulin and thyroglobulin antibodies in differentiated thyroid cancer patients. Horm Metab Res. 2010:42:736-9.

22. Elzein E, Zablocki J. A1 adenosine receptor agonists and their potential therapeutic applications. Expert Opin Investig Drugs. 2008:17:1901-10.

23. Haskó G, Linden J, Cronstein B, Pacher P. Adenosine receptors: therapeutic aspects for inflammatory and immune diseases. Nat Rev Drug Discov. 2008;7:759-70

24. Cristalli G, Müller CE, Volpini R. Recent developments in adenosine A2A receptor ligands. Handb Exp Pharmacol. 2009;193:59-98.

25. Feoktistov I, Biaggioni I. Role of adenosine A2B receptors in inflammation. Adv Pharmacol. 2011;61:115-44

26. Koeppen M, Eckle T, Eltzschig HK. Interplay of hypoxia and A2B adenosine receptors in tissue protection. Adv Pharmacol. 2011;61:145-86. 
27. Fishman P, Bar-Yehuda S, Liang BT, Jacobson KA. Pharmacological and therapeutic effects of A3 adenosine receptor agonists. Drug Discov Today. 2012;17:359-66.

28. Fishman P, Bar-Yehuda S, Synowitz M, Powell JD, Klotz KN, Gessi S, et al. Adenosine receptors and cancer. Handb Exp Pharmacol. 2009;193:399-441.

29. Fishman P, Bar-Yehuda S, Ohana G, Pathak S, Wasserman L, Barer F, et al. Adenosine acts as an inhibitor of lymphoma cell growth: a major role for the A3 adenosine receptor. Eur J Cancer. 2000;36:1452-8.

30. Spychal J. Tumor-promoting functions of adenosine. Pharmacol Ther 2000;87:161-73.

31. Mujoomdar M, Bennett A, Hoskin D, Blay J. Adenosine stimulation of proliferation of breast carcinoma cell lines: evaluation of the [3H] thymidine assay system and modulatory effects of the cellular microenvironment in vitro. J Cell Physiol. 2004;201:429-38.

32. Yamano Y, Uzawa K, Shinozuka K, Fushimi K, Ishigami T, Nomura H, et al Hyaluronan-mediated motility: a target in oral squamous cell carcinoma. Int J Oncol. 2008;32:1001-9.

33. Shimizu F, Shiiba M, Ogawara K, Kimura R, Minakawa Y, Baba T, et al. Overexpression of LIM and SH3 protein 1 leading to accelerated G2/M phase transition contributes to enhanced tumourigenesis in oral cancer. PLoS One. 2013;8:e83187.

34. Wei Q, Costanzi S, Balasubramanian R, Gao ZG, Jacobson KA. A2B adenosine receptor blockade inhibits growth of prostate cancer cells. Purinergic Signal. 2013;9:271-80.

35. Merighi S, Mirandola P, Milani D, Varani K, Gessi S, Klotz KN, et al. Adenosine receptors as mediators of both cell proliferation and cell death of cultured human melanoma cells. J Invest Dermatol. 2002;119:923-33.

36. Semenza GL. Targeting HIF-1 for cancer therapy. Nat Rev Cancer. 2003;3:721-32.

37. Dammen R, Haugen M, Svejda B, Alaimo D, Brenna O, Pfragner R, et al. The stimulatory adenosine receptor ADORA2B regulates serotonin (5-HT) synthesis and release in oxygen-depleted EC cells in inflammatory bowel disease. PLoS One. 2013;8, e62607.

38. Ning C, Wen J, Zhang Y, Dai Y, Wang W, Zhang W, et al. Excess adenosine A2B receptor signaling contributes to priapism through HIF-1 a mediated reduction of PDE5 gene expression. FASEB J. 2014;28:2725-35.

39. Merighi S, Simioni C, Gessi S, Varani K, Mirandola P, Tabrizi MA, et al. A(2B) and $A(3)$ adenosine receptors modulate vascular endothelial growth factor and interleukin-8 expression in human melanoma cells treated with etoposide and doxorubicin. Neoplasia. 2009;11:1064-73.

\section{Submit your next manuscript to BioMed Central and take full advantage of:}

- Convenient online submission

- Thorough peer review

- No space constraints or color figure charges

- Immediate publication on acceptance

- Inclusion in PubMed, CAS, Scopus and Google Scholar

- Research which is freely available for redistribution 